\title{
Delay difference equations: Coexistence of oscillatory and nonoscillatory solutions
}

\author{
Ravi P. Agarwal, Martin Bohner*, José M. Ferreira, Sandra Pinelas
}

Received: September 14, 2013

Summary: The aim of this work is to study the coexistence of oscillatory and nonoscillatory solutions of a certain scalar delay difference equation.

\section{Introduction}

The aim of this work is to study the simultaneous existence of oscillatory and nonoscillatory solutions of the linear delay difference equation

$$
\begin{aligned}
& x(t)=\sum_{j=1}^{p} a_{j}(t) x\left(t-r_{j}\right), \quad t>0 \\
& x(t)=\phi(t), \quad-r_{p} \leq t \leq 0,
\end{aligned}
$$

where $x:\left[-r_{p}, \infty\right) \rightarrow \mathbb{R}, 0<r_{1}<r_{2}<\ldots<r_{p}, a_{j} \in \mathrm{C}([0, \infty), \mathbb{R})$ for all $j \in\{1,2, \ldots p\}$, and $\phi \in \mathrm{C}\left(\left[-r_{p}, 0\right], \mathbb{R}\right)$ is such that

$$
\phi(0)=\sum_{j=1}^{p} a_{j}(0) \phi\left(-r_{j}\right) .
$$

By a solution of (1.1)-(1.2) we mean a function $x \in \mathrm{C}\left(\left[-r_{p}, \infty\right), \mathbb{R}\right)$ that satisfies (1.1)(1.2). Such a solution is called oscillatory if it has arbitrarily large zeros; otherwise, it is said to be nonoscillatory. For the discrete version (1.1), oscillation theory has been developed mainly in the last two decades. For example, see [1-4, 10], and for some recent works see $[5,7]$ and the references cited therein. The oscillatory and nonoscillatory behavior of solutions of (1.1) has been relatively less studied. For the autonomous case of $(1.1)$, we refer to $[8,9]$ and the references cited therein.

In this paper, we shall examine the coexistence of both oscillatory and nonoscillatory solutions of (1.1). In Section 2, under the assumptions on the delays given above, we shall

* Corresponding author: Martin Bohner

AMS 2010 subject classification: Primary: 39A10, 39A12, 39A21

Key words and phrases: Difference equations, oscillations, nonoscillations 